\title{
The use of polyethylene glycol hydrogel tissue adhesive for corneal incision closure following Descemet membrane endothelial keratoplasty (DMEK)
}

\author{
Morgan Fredell ${ }^{1} \cdot$ Marshall B. Hamill $^{2}$ \\ Received: 1 October 2018 / Accepted: 29 December 2018 / Published online: 6 February 2019 \\ (c) The Royal College of Ophthalmologists 2019
}

The most frequent complication in Descemet membrane endothelial keratoplasty (DMEK) is partial or complete graft detachment of the donor material from the host cornea [1]. Maintenance of an anterior chamber gas bubble through good wound closure is critical to ensure graft adherence [1]. Currently, suturing the main incision is recommended to avoid loss of the gas bubble to minimize graft detachment [2]. While sutures are the traditional method for closing corneal incisions [3], sutureless closure is gaining popularity as closure with adhesives can result in a better watertight seal [3, 4].

For polyethylene glycol hydrogel tissue adhesive (PEG) to be successful for DMEK closure, the closed wounds must withstand the high intra-ocular pressures (IOPs) necessary for graft adherence postoperatively and after rebubbling to prevent the loss of gas and must not increase graft detachament rates, which to our knowledge, no study has investigated. This study analyzes DMEK surgeries closed with PEG to assess maintenance of wound closure immediately postoperatively and during rebubbling and to assess detachment rates to determine if PEG should be considered an alternative to sutures.

Retrospective chart review identified 48 consecutive cases of DMEK closed with PEG (ReSure Sealant, Ocular Therapeutix, Inc., Bedford, Massachusetts, USA) performed by one surgeon between 11 May 2016 and 8 March 2017 at Cullen Eye Institute, Baylor College of Medicine. Mean age of the patients was $71.1 \pm 8.5$ [49.0-90.0] and 15 of the

Marshall B. Hamill

Mhamill@bcm.edu

1 School of Medicine, Baylor College of Medicine, 6501 Fannin, NC-200, Houston, TX 77030, USA

2 Department of Ophthalmology, Baylor College of Medicine, 6501 Fannin, NC-200, Houston, TX 77030, USA
Table 1 Intraocular Pressures post DMEK and Rebubbling

\begin{tabular}{llll}
\hline Intraocular pressures $^{\mathrm{a}}$ & Mean (SD) & Minimum & Maximum \\
\hline $\begin{array}{l}\text { Intraocular pressure (mmHg) at: } \\
\quad \text { End of surgery }\end{array}$ & $13.9(4.8)$ & 3.0 & 27.2 \\
$\quad 18.2(6.3)$ & 8.0 & 37.0 \\
$\quad$ Day 1 post-op & $14.6(4.2)$ & 7.0 & 32.0 \\
$\quad$ Week 1 post-op ( $n=47)$ & $20.3(4.2)$ & 17.0 & 25.0 \\
$\quad \begin{array}{l}\text { Immediately post rebubbling } \\
(n=3)\end{array}$ & $12.8(2.9)$ & 10.0 & 17.0 \\
$\quad \begin{array}{l}\text { Day 1 post rebubbling } \\
(n=6)\end{array}$ & $6.2(1.5)$ & 5.0 & 8.0 \\
$\begin{array}{l}\text { Time from surgery to } \\
\text { rebubbling (days) }\end{array}$ & & & \\
\hline
\end{tabular}

Intraocular pressures were measured by Schiotz tonometry at the conclusion of the DMEK procedure and at day 1 post-op, and week 1 post-op. Intraocular pressures were measured by Tono-Pen ${ }^{\circledR}$ (Reichert Inc., Buffalo, New York, USA) immediately following the rebubbling procedure and at day 1 post rebubbling

${ }^{a}$ Includes only patients rebubbled at $\leq 8$ days post-op. Two patients were excluded who were rebubbled at 20 and 28 days post-op.

cases were combined with phacoemulsification + IOL. This study was approved by the Institutional Review Board.

Surgeries were performed according to a standard protocol, which included corneal incisions of $2.5 \mathrm{~mm}$ in length. The host was stripped to the same size or $1 / 2 \mathrm{~mm}$ larger than the donor with donor diameters ranging from 7.5 to $8.0 \mathrm{~mm}$. Donor material was prepared by the Eye Bank and inserted via a glass pipette. The incision was closed with a single application of PEG. Then the donor scroll was unrolled utilizing the "tapping" technique [5]. At the end of the case, the anterior chamber was filled to $80 \%$ bubble by volume with $20 \%$ SF6 gas. Patients were rebubbled at the slit lamp with air injected through the inferior surgical paracentesis if graft separation of greater than $25 \%$ of the donor disc was noted at the 1-week postoperative visit. 
PEG-closed wounds withstood a maximum IOP of $27.2 \mathrm{mmHg}$ immediately postoperatively and $25 \mathrm{mmHg}$ immediately post rebubbling (Table 1). A water tight seal was maintained following initial surgery with only a single application of PEG and after rebubbling without further PEG application. No instance of wound failure or leakage occurred. The detachment rate was $12.5 \%$ with six eyes undergoing rebubbling.

This study contributes to the literature of sutureless closure of corneal incisions. As the PEG-closed incisions did not experience any leakage and allowed sufficient wound healing to prevent the wound reopening during rebubbling, we conclude that PEG should be considered as an alternative to sutures. This is further supported as the detachment rate in this study is less than or similar to published rates [2]. This conclusion is limited in validity due to the observational retrospective nature of the study methods.

Funding: Funding was provided through an unrestricted grant from the Hamill Foundation, Houston, Texas, USA.

\section{Compliance with ethical standards}

Conflict of interest The authors declare that they have no conflict of interest.

Publisher's note: Springer Nature remains neutral with regard to jurisdictional claims in published maps and institutional affiliations.

\section{References}

1. Dirisamer M, van Dijk K, Dapena I, Ham L, Oganes O, Frank LE, et al. Prevention and management of graft detachment in Descemet membrane endothelial keratoplasty. Arch Ophthalmol. 2012;130:280-91.

2. Tan DTH, Ang M. Surgical techniques for DSEK. (Mannis MJ, Holland EJ, eds). Cornea: Fundamentals, Diagnosis, and Management. 4th edn, p. 1438-79. St. Louis: Elsevier Inc; 2016.

3. Matossian C, Makari S, Potvin R. Cataract surgery and methods of wound closure: a review. Clin Ophthalmol. 2015;9:921-8.

4. Uy HS, Kenyon KR. Surgical outcomes after application of a liquid adhesive ocular bandage to clear corneal incisions during cataract surgery. J Cataract Refract Surg. 2013;39:1668-74.

5. Yoeruek E, Bayyoud T, Hofmann J, Bartz-Schmidt K-U. Novel maneuver facilitating Descemet membrane unfolding in the anterior chamber. Cornea. 2013;32:370-3. 